Journal of Animal and Veterinary Advances 11 (2): 218-223, 2012

ISSN: $1680-5593$

(C) Medwell Journals, 2012

\title{
A Novel and Reasonable Approach for in vivo Liver Gene Transfection in Rats
}

\author{
Wang Baolin, Yuan Tao, Liu Menggang, Chen Hongxu and Chen Ping \\ Department of Hepatobiliary Surgery, Research Institute of Surgery, Daping Hospital, \\ Third Military Medical University, Chongqing, China
}

\begin{abstract}
The hepatocellular gene transfection in vitro has been repeatedly reported but the liver gene transfection in vivo is still poorly understood. The liver gene transfection can be performed through injection via tail vein and portal vein but these methods result easily bleeding or stress reaction to the animal which may potentially confound experimental results. Here to exploring a reasonable and effective injection method for liver gene transfection, recombinant lentiviral vectors with Green Fluorescent Protein gene (GFP) were injected, respectively via the rat's cecal vein, portal vein and tail vein and then it was observed that the GFP expression of the liver tissue and the effect of various injections on the rats. The results showed that via the cecal vein injection had the high transfection efficiency and little increase of portal vein press and the minimum effect on liver transaminase and serum IL-6 and TNF- $\alpha(\mathrm{n}=20)(\mathrm{p}<0.001)$. It was demonstrated that in vivo liver gene transfection via the cecal vein injection in rats is a novel and reasonable method.
\end{abstract}

Key words: Liver gene transfer, cecal vein, lentiviral vectors, rats, injection, China

\section{INTRODUCTION}

Liver is an interesting organ that undertakes many complex and important functions for the organism (Atta, 2010). The research on liver has entered into the gene level so the exogenous genes often need to be transferred into the hepatocytes.

The technique in vitro the hepatocellular gene transfection has been repeatedly reported and has become mature. But the liver gene transfection in vivo is still poorly understood. Currently, injection of the liver gene transfection is performed via tail vein in mice (Maruyama et al., 2004) but a lot of viral plasmids need to be hydrodynamic injected in the experiment which may overload the physiological quantity and even lead to the mice death, meanwhile the transfection efficiency is low.

The injection via portal vein (Follenzi et al., 2002) in rats is generally performed to acquire liver gene transfection in vivo, so the virus injection quantity can be reduced and the transfection efficiency is obviously better than via tail vein. But the position of the rat portal vein is quite deep to result the rat death due to easy bleeding then it lead to the failure of experiment. More importantly that the injection via portal vein results in the changes of hepatic blood flow and the pressure may cause the secretion of various cell factors by the liver sinusoidal cells (Ping et al., 2006) thereby changing internal environment of liver and may potentially confounding experimental results. It seems that how to reach the best transfection efficiency, minimize the interference factors in the experiment and improve the success rate of experiment has become a very tricky contradiction. But how can we solve this contradiction in the experiment?

Researchers observed in the experiment that the rat's cecum was obviously more enlargement than the nearby colon and small intestine and was located at the right lower quadrant with a superficial position, equivalently slightly above the human McBurney point. More importantly, the rat's cecal vein was also quite obvious (Fig. 1).

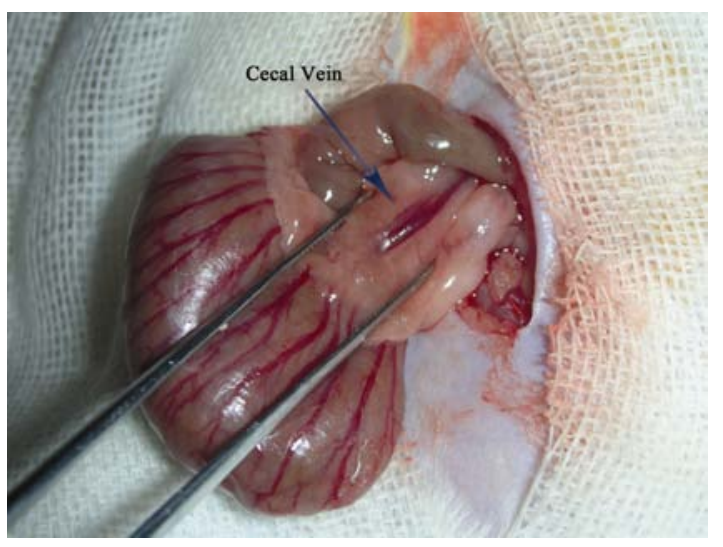

Fig. 1: The quite obvious cecal vein in the mesocecum

Corresponding Author: Chen Ping, 10 Changjiangzhilu Daping, 400042 Chongqing, P.R. China 
The cecal vein belonged to the branch of portal vein which might be a good access for performing the liver gene transfection. In the present research, researchers injected lentiviral vectors with Green Fluorescent Protein gene (GFP) via cecal vein to perform the liver gene transfection in rat and then observed the GFP expression of the liver tissue section.

The results show that the transfection efficiency via the cecal vein is almost consistent with that via the portal vein but the success rate of operation of injection via the cecal vein is higher and it has minimum effect on the rats. Researchers speculate that the injection via cecal vein may be the best approach for the liver gene transfection and the cecal vein approach may be selectively used in the other liver-related study and clinical gene therapy.

\section{MATERIALS AND METHODS}

Lentiviral vectors: Lentiviral vectors with GFP gene was constructed by the transient calcium phosphate cotransfection of $293 \mathrm{~T}$ cells as previously described (Naldini et al., 1996; Giannini et al., 2003). The vector supernatant was collected by centrifugation $(100,000 \mathrm{~g}$ for $1 \mathrm{~h}$ at $4^{\circ} \mathrm{C}$ ) and stored at $-80^{\circ} \mathrm{C}$ until use.

Rats and study design: Male Sprague-Dawly rat (200 300 g) were used (Experimental Animal Center, Third Military Medical University, Chongqing, China) with the approval of the Animal Care Committee of The Third Military Medical University. Animals were housed in the animal facility under Specific Pathogen Free (SPF) conditions with a $12 \mathrm{~h}$ light-to-dark cycle and free access to food and water. Rats received humane care in compliance with the guide for the care and use of laboratory animals (ILAR, 1996).

Researchers carried out the experiment in 85 rats in total. Initially, researchers used 20 rats to verify the feasibility of injection via cecal vein for the liver gene transfection in vivo and then used 65 rats to compare the advantages of via cecal vein, among which 20 rats were respectively used as the Cecal Vein group (CV), Portal Vein group (PV) and Tail Vein group (TV) and the remaining 5 rats were used as a control group.

Injection preparation and operation: After 2\% pentobarbital sodium parenteral solution was injected into the abdominal cavity ( $45 \mathrm{mg} \mathrm{kg}^{-1}$ ) and then the rat was completely narcotized and was fixed on the platform in a supine position, observed the fluctuate of the rat thorax to detect both cardiac and respiratory waveforms. Then, researchers used a depilatory to remove the hair from the abdominal cut as previously described (Blair-Handon et al., 2010) and then iodophors for disinfection, cover sterile gauze with a hole. The researchers took about $1 \mathrm{~cm}$ incision from the right lower quadrant (equivalently slightly above the McBurney point) for the cecal vein group. After opening the abdominal cavity, researchers see a grossus cecum easily, lightly raise it and turn it over to see an obvious cecal vein in the mesocecum. Slowly inject $1 \mathrm{~mL}$ GFP-lentiviral vector solution (about $3 \times 10^{7} \mathrm{TU}$ ) with the microinjector (Hamilton Bonaduz, $\mathrm{AG}$ ) via cecal vein and needle tip towards liver (Fig. 1).

The injection be performed, researchers lightly pressed the cecal vein towards the nearby fat pad about $3 \mathrm{~min}$, sutured the abdominal wall and then disinfected it. The injection via portal vein (Follenzi et al., 2002) and via tail vein (Maruyama et al., 2004) can be performed, respectively as previously described. The remaining five rats was used as controls, their abdominal cavity was opened and closed immediately without injection. Before the operation, the rat must be completely unconscious. During the operation, the attention should be paid to sterile operation to reduce the risk of postoperative infection and a small amount of normal saline can be used for moistening the cecum. After the operation, the rats should be given the humane care such as warming and normal food and water intake.

Measurement of portal vein pressure: To assess the effect of injections via the portal vein and cecal vein on the portal vein pressure, researchers carried out injection via portal vein and via cecal vein in three rats, respectively and performed measurement of portal vein pressure with PowerLab data acquisition systems/8 sp ( $\mathrm{AD}$ instruments $\mathrm{AU}$ ) during the injection. By taking the method as above mentioned after the rat was narcotized, fixed and sterilized, the abdominal cavity was opened to fully display the portal vein, PowerLab/ $8 \mathrm{sp}$ artery built-in pipe was distally inserted into the portal vein and then fixed well and then $1 \mathrm{~mL}$ normal saline was injected via the cecal vein or the portal vein and simultaneously, the changes of portal vein pressure were recorded.

Microscopic analyses: Within 2 days after injection, the rats liver tissue is fetched out and cut into $10 \mu \mathrm{m}$ thin sections after the quick frozen $\left(30 \mathrm{~min}\right.$ at $\left.-25^{\circ} \mathrm{C}\right)$ and the section was fixed on the microscope slide. The light should be avoided during the operation process. The expression of green fluorescence was rapidly observed with laser scanning confocal microscope.

Assessment of the influence of the rats: To assess liver function and inflammation of stress reaction, researchers measured the Alanine aminotransferase (ALT) and Aspartate Transaminase (AST) and Tumor Necrosis Factor- $\alpha$ (TNF- $\alpha$ ) and Interleukin-6 (IL-6) of blood in the portal vein of 65 rats. The serum was collected by centrifugation $\left(1,200 \mathrm{~g}\right.$ for $10 \mathrm{~min}$ at $\left.20^{\circ} \mathrm{C}\right)$ and then was measured according to the manufacturer's protocol of the 
Enzyme-Linked Immunosorbent Assay (ELISA) commercial kit (eBioscience, San Diego, CA). Each sample was measured at least three times. Briefly, samples were added to the appropriate wells of precoated ELISA plates and incubated at $37^{\circ} \mathrm{C}$ for $1 \mathrm{~h}$. Plates were rinsed and detection antibodies were added. After washing, substrate solution was added. The plates were read at $450 \mathrm{~nm}$ in an ELISA reader.

Statistical analysis: Statistics analyses were performed by SPSS 13.0 software (SPSS Inc., Chicago, IL, USA) and the results were presented as mean $\pm \mathrm{SD}$. Comparison of means was performed by one-way ANOVA (Scheffe as post-hoc test), $\mathrm{p}<0.05$ was considered significant.

\section{RESULTS AND DISCUSSION}

GFP expression was observed in both cecal vein and portal vein group and obviously better than tail vein group: Comparison with the tail vein group, obvious green fluorescent be observed in both cecal vein and portal vein group.

The sections of liver tissue has a strong green fluorescence expression (Fig. 2a, b) the green fluorescence was expressed on the hepatic cord and the transfection rate reaches $>70 \%$ but green fluorescent was only scattered in tail vein group (Fig. 2c) and no green fluorescence is seen from the section of the control group (Fig. 2d)

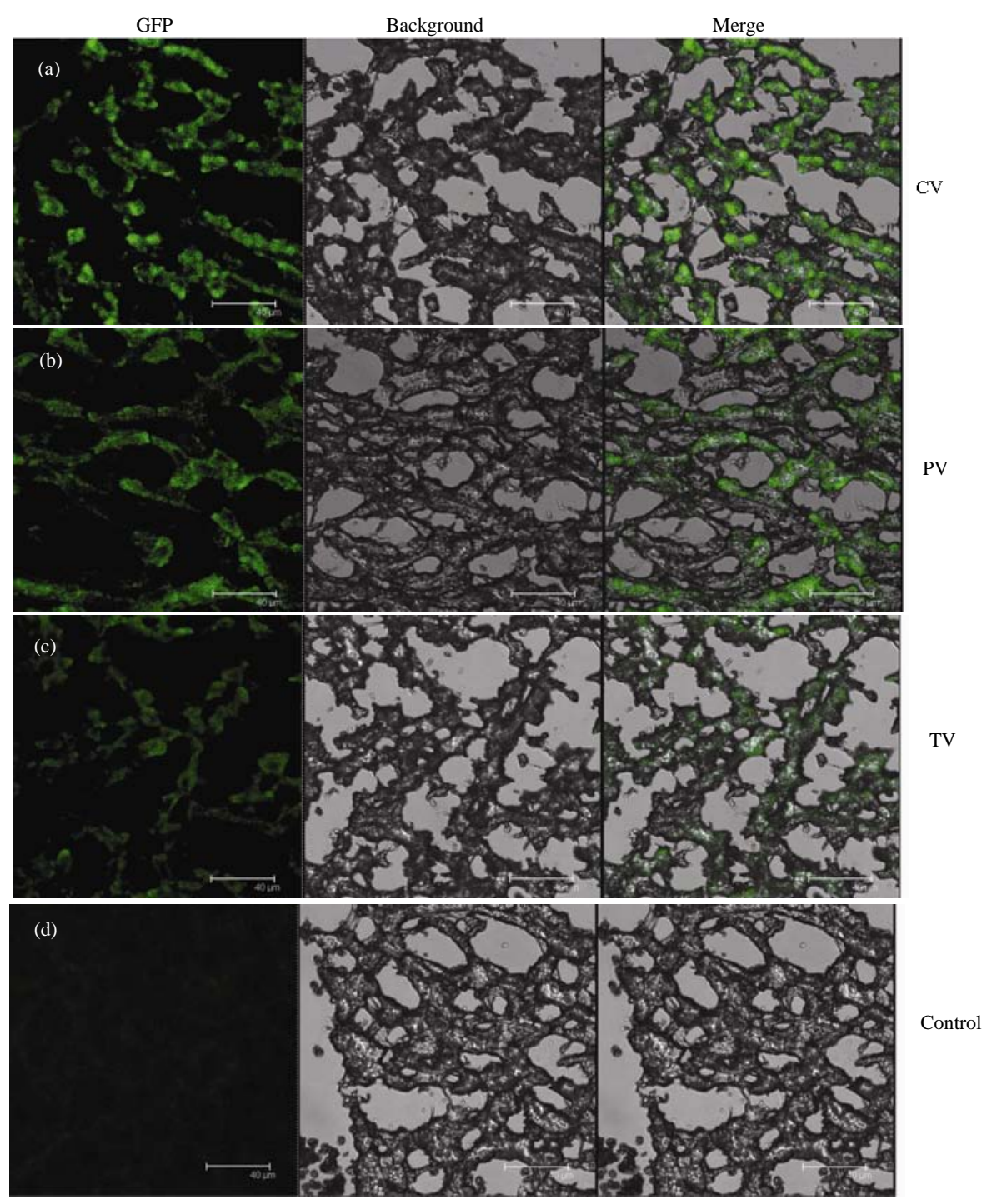

Fig. 2: The green fluorescence is observed with laser scanning confocal microscope from the cryosections of live; a) is the Cecal Vein (CV); b) is the Portal Vein (PV); c) is the Tail Vein (TV) and d) is the control group. The green fluorescence which is demonstrated in the merge group is expressed on the hepatic cell cords. CV is basically consistent with PV and obviously higher than TV but the control group has no fluorescence expression 

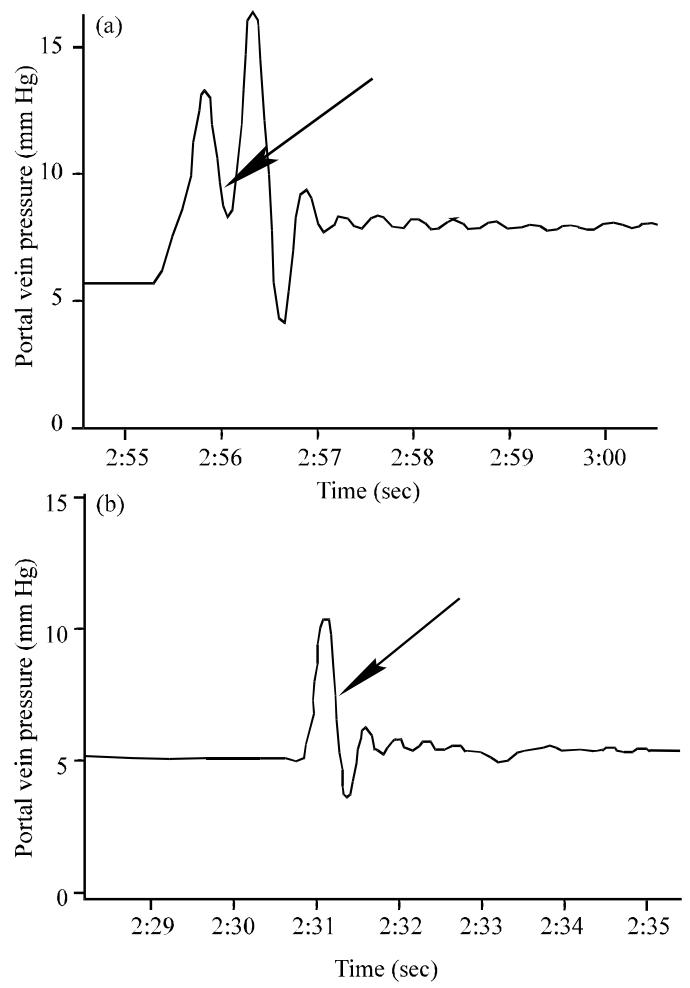

Fig. 3: Change curve of portal vein pressure; a) the portal vein pressure obviously increases during the injection via portal veil and the pressure increases by about $3 \mathrm{~mm} \mathrm{Hg}$ at a certain time after the injection begins (as shown by the arrow) and $b$ ) the injection via cecal vein only results in the instantaneous fluctuation of portal vein pressure (as shown by the arrow) and the pressure can be returned to the normal level very soon

The injection via the portal vein increased portal vein pressure but via the cecal vein has little effect: The injection via portal vein increased the pressure of portal vein about $3 \mathrm{mmHg}$ (Fig. 3).

The pressure curve of PowerLab/8 sp obviously indicated that the injection via cecal vein had little effect on portal vein pressure and the instantaneous fluctuation of portal vein pressure may be caused only in the operation but the pressure can be returned to a normal level very soon.

The cecal vein group had the minimum effect on liver function: Serum transaminase level of the cecal vein group was obviously lower than the portal vein group (ALT: $182.0 \pm 29.9 \mathrm{IUL}^{-1}$ vs. $391.4 \pm 50.0 \mathrm{IU} \mathrm{L}^{-1}, \mathrm{p}<0.001$; AST: $114.4 \pm 17.5 \mathrm{IU} \mathrm{L}^{-1}$ vs. $235.6 \pm 36.2 \mathrm{IU} \mathrm{L}^{-1}, \mathrm{p}<0.001$; Fig. 4a, b). The ALT level of portal vein group and tail vein group had statistically significant differences but the
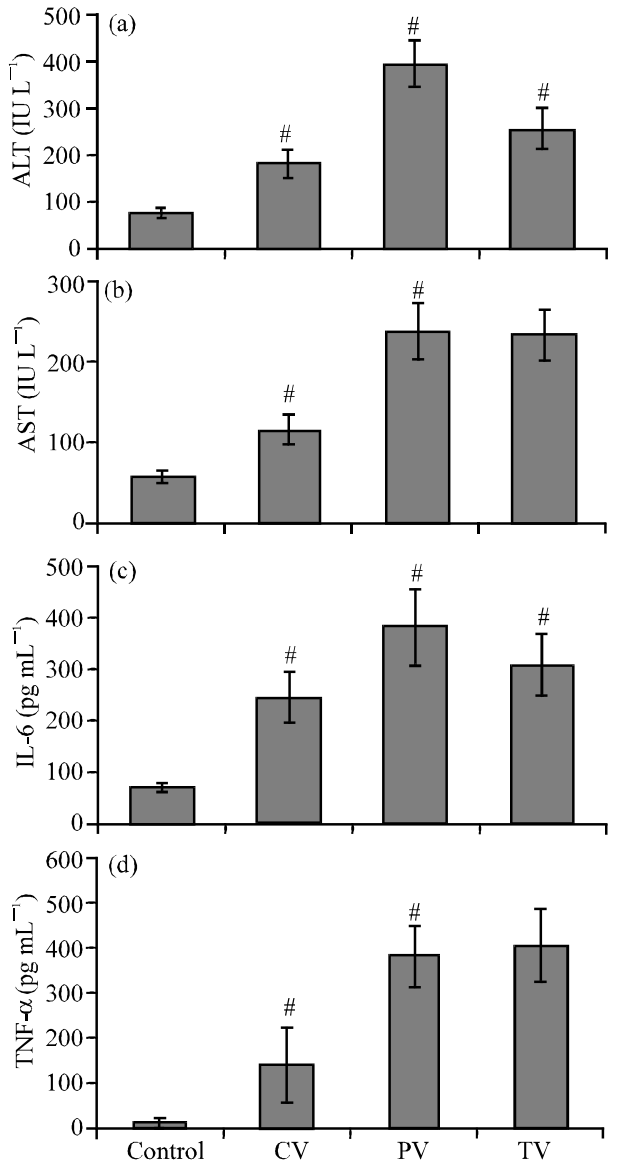

Fig. 4: The levels of serum transaminase and IL-6 and $\mathrm{TNF}-\alpha$. Within 2 days after injection, each sample was measured at least three times to gain the average level $(\mathrm{n}=5$ for the control group and $\mathrm{n}=20$ for the remaining three groups); $\mathrm{a}, \mathrm{b}$ ) The ALT and AST levels of cecal vein group are obviously lower than those of portal vein group and tail vein group $(\# p<0.001)$ and the difference of AST level between portal vein group and tail vein group is not obvious and c, d) the IL-6 and TNF- $\alpha$ levels of cecal vein group are obviously lower than those of portal vein group and tail vein group (\#p<0.001) but the TNF- $\alpha$ level of tail vein group was slightly higher than that portal vein group

AST levels were similar without significant differences. The results of the treatment groups had significant differences from that of the control group $(\mathrm{p}<0.001)$.

The cecal vein group had the minimum inflammatory reaction: Similarly to assess inflammatory reaction of the rats, researchers measured the TNF- $\alpha$ and IL- 6 of the all 
rats. The IL- 6 and TNF- $\alpha$ levels of the cecal vein group was lower than the portal vein group (IL-6: $244.5 \pm 48.4 \mathrm{pg} \mathrm{mL}^{-1}$ vs. $378.5 \pm 73.6 \mathrm{pg} \mathrm{m} \mathrm{L}^{-1}, \mathrm{p}<0.001$; TNF- $\alpha$ : $137.4 \pm 81.8 \mathrm{pg} \mathrm{m} \mathrm{L}^{-1}$ vs. $380.9 \pm 69.4 \mathrm{pg} \mathrm{m} \mathrm{L}^{-1}$, $\mathrm{p}<0.001$; Fig. 4c, d) and the tail vein group (IL-6307.1 \pm 61.5 pg m L ${ }^{-1}, \mathrm{TNF}-\alpha 404.8 \pm 80.4 \mathrm{pg} \mathrm{m} \mathrm{L}^{-1}$ ). The IL-6 levels of portal vein and tail vein had statistically significant differences $(p<0.001)$ but the TNF- $\alpha$ level of tail vein group was slightly higher than that of portal vein group and the differences had no statistical significance.

\section{DISCUSSION}

The gene therapy is an important development direction for the treatment of liver diseases and especially, the tumor diseases. Various target genes and interference genes can be transferred into the liver in vivo by lentiviral vectors (Lois et al., 2002) to reach gene correction and these genes can be detected by GFP probe.

But it still need further research that how to more conveniently and reasonably transfer the exogenous therapeutic genes into the liver how to understand the immune response of lentiviral vectors in the gene transfection and the long-term expression of transgene, etc. (Sandig and Strauss, 1996; Nguyen and Ferry, 2004). But if we found a reasonably transfection approach, this will provide more help for the subsequent experiments in vivo. As all knows, the blood of liver comes from both portal vein and hepatic artery and the portal vein accounts for $70 \sim 75 \%$ of liver blood supply. The portal vein system is situated among both blood capillaries nets and relatively independent of the systemic circulation so, the injection via the portal vein system more target towards the liver and thus the portal vein system is often used for injection of liver gene transfection. The cecal vein belongs to one branches of the portal vein system. Researchers experimentally obtained the well transfection of liver gene via cecal vein injection. The gene transfection efficiency via cecal vein was consistent with that via portal vein. What needs to be pointed out is that the injection via the cecal vein as an easier and safer method for liver gene transfection than injection via the portal vein.

The rat's cecal vein is also obvious and easy to be found and there are rich adipose tissues around it (Fig. 1) which is favorable for hemostasis after injection. But the position of hepatic portal vein was very deep and many important blood vessels were also around it. Two rats accidentally died due to bleeding in abdominal cavity in ours portal vein group (the SD rats of the same standard were immediately used). In short, the injection via cecal vein is a safer operation way.

Meanwhile, the results indicated that the serum transaminase in the cecal vein group was lower than that in the portal vein and the tail vein group. The injection via cecal vein could not result in continuous portal hypertension, so more stress reaction factors would not be produced due to stimulation which was verified by the levels of IL- 6 and TNF- $\alpha$. As the injection via portal vein resulted in continuous portal hypertension, various cell factors were secreted in the blood (Ping et al., 2006) including IL- 6 and $\mathrm{TNF}-\alpha$. The injection via tail veil induced the increases of IL- 6 and TNF- $\alpha$ which partly because the viruses enter into the circulation system or stimulate factors such as pain. These stress reaction factors may also change the liver internal environment in vivo and thus, potentially confound experimental results which may be reduced via cecal vein injection.

\section{CONCLUSION}

The injection via the rat cecal vein for the liver gene got better transfection effect and has little stimulation to the rat. The cecal vein is selected in the rat's liver gene study in vivo so that the experiment will be more convenient and accurate. If the experiment is carried out the smaller animals like mice, the cecal vein be too thin should be excluded. Furthermore, researchers believe that the injection approach via cecal vein will play a more role in the future research in rats.

\section{ACKNOWLEDGEMENTS}

This study was supported by the National Natural Science Foundation of China (grants 30972895) and the Natural Science Foundation of Chongqing of China (CSTC 2009BA5014).

\section{REFERENCES}

Atta, H.M., 2010. Gene therapy for liver regeneration: Experimental studies and prospects for clinical trials. World J. Gastroenterol., 16: 4019-4030.

Blair-Handon, R., K. Mueller and S. Hoogstraten-Miller, 2010. An alternative method for intrathymic injections in mice. Lab Anim., 39: 248-252.

Follenzi, A., G. Sabatino, A. Lombardo, C. Boccaccio and L. Naldini, 2002. Efficient gene delivery and targeted expression to hepatocytes in vivo by improved lentiviral vectors. Human Gene Ther., 13: 243-260. 
Giannini, C., S. Morosan, J.G. Tralhao, J.E. Guidotti and. Battaglia et al., 2003. A highly efficient, stable and rapid approach for $E x$ vivo human liver gene therapy via a FLAP lentiviral vector. Hepatology, 38: 114-122.

ILAR, 1996. Guide for the Care and Use of Laboratory Animals. National Academies Press, Washington, DC.

Lois, C., E.J. Hong, S. Pease, E.J. Brown and D. Baltimore, 2002. Germline transmission and tissue-specific expression of transgenes delivered by lentiviral vectors. Science, 295: 868-872.

Maruyama, H., N. Higuchi, S. Kameda, J.I. Miyazaki and F. Gejyo, 2004. Rat liver-targeted naked plasmid DNA transfer by tail vein injection. Mol. Biotechnol., 26: 165-172.
Naldini, L., U. Blomer, P. Gallay, D. Ory and R. Mulligan et al., 1996. In vivo gene delivery and stable transduction of nondividing cells by a lentiviral vector. Science, 272: 263-267.

Nguyen, T.H. and N. Ferry, 2004. Liver gene therapy: Advances and hurdles. Gene Therapy, 11: S76-S84.

Ping, C., D. Xiaoling, Z. Jin, D. Jiahong, D. Jiming and Z. Lin, 2006. Hepatic sinusoidal endothelial cells promote hepatocyte proliferation early after partial hepatectomy in rats. Arch. Med. Res., 37: $576-583$.

Sandig, V. and M. Strauss, 1996. Liver-directed gene transfer and application to therapy. J. Mol. Med., 74: 205-212. 